\title{
Physical and chemical stability of triethylenetetramine dihydrochloride, an API against Wilson's disease
}

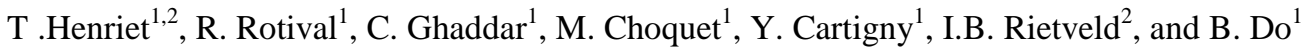 \\ ${ }^{1}$ Départements Laboratoires, Etablissement Pharmaceutique des Hôpitaux de Paris, 75005 Paris, France \\ ${ }^{2}$ Laboratoires Physico-chimie Industrielle du Médicament, EA 4066, Faculté des Sciences Pharmaceutiques et Biologiques, \\ Université Paris-Descartes, 75006 Paris, France \\ ${ }^{3}$ Laboratoires des Sciences et Méthodes Séparatives, EA 3233, UFR Sciences et Techniques, Université de Rouen, 76821 \\ Mont Saint Aignan, France
}

The stability behavior of an active pharmaceutical ingredient (API) is important for the development of a reliable drug formulation. Triethylenetetramine dihydrochloride is an API used as second-line treatment of Wilson's disease since the early 1980's. Nonetheless, a comprehensive understanding of its stability behavior is lacking. In this work, its stability has been determined both in the solid state and in aqueous solution, thus evaluating on the one hand the relative stability of polymorphs and hydrates and, on the other hand, the main decomposition products and their pathways.

For the solid-state study, the API was exposed to several conditions involving temperature and moisture content. Thermal analysis and dynamic vapor sorption were used to obtain information about the physical stability. Liquid chromatography-tandem mass spectrometry (LCMS) was used to study the chemical decomposition. In addition, solutions of the API in water were exposed to heat, acid, alkaline and oxidative conditions, so-called stress tests, followed by LC-MS measurements.

Solid-state analyses demonstrated the existence of two polymorphs and a dihydrate, as had been reported previously [1, 2]. The dimorphism was found to be monotropic under ordinary conditions using the results from the thermal analysis in combination with the Ostwald rule and the Le Chatelier principle; the commercial form was the most stable of the two forms. In addition, the relative-humidity domain in which the dihydrate is stable has been assessed.

Two compounds derived from triethylenetetramine were identified by LC-MS after the stress tests in solution. These compounds are found in very low levels in native samples too and might therefore be degradation products as well as impurities caused by synthesis.

\section{References}

1. Philera New Zealand limited (NZ), Synthesis of triethylenetetramines, M. Jonas et al., US Patent, US8067641 (2011)

2. C.A. Ilioudis et al., New Journal of Chemistry 24(10) (2000) 